\title{
Toward generalized planetary stereo analysis scheme-Prototype implementation with multi-resolution Martian stereo imagery
}

\author{
Jung-Rack Kim ${ }^{1}$, Shih-Yuan Lin $^{2}$, Yun-Soo Choi ${ }^{1}$, and Young-Hwi Kim ${ }^{3}$ \\ ${ }^{1}$ Department of Geoinformatics, University of Seoul, Seoulsiripdaero 163, Dongdaemum-gu, Seoul 130-743, Korea \\ ${ }^{2}$ Department of Land Economics, National Chengchi University, 64, Sec. 2, Zhinan Rd., Wenshan Dist., Taipei 11605, Taiwan \\ ${ }^{3}$ Department of Computer Science, Yonsei University, Shinchoen-dong, Seadeamun-gu, Seoul 120-749, Korea
}

(Received January 31, 2012; Revised August 16, 2012; Accepted November 23, 2012; Online published August 23, 2013)

\begin{abstract}
Stereo analysis of orbital imagery is highly valuable for scientific research in planetary surface. Thus, the processing of planetary stereo imagery has been progressed with various approaches and resulted in a series of uncontrolled topographic products. In order to fully utilize the data derived from image systems carried on various planetary orbiters, the generalized algorithms of stereo image processing and Digital Terrain Model (DTM) extraction have been developed. Based on Kim and Muller's approach (2009), the algorithms were updated employing the feed-forwarded model-based matcher and the generic sensor model. It is a sort of iterative stereo procedure delivering the reference data to next stage for $3 \mathrm{D}$ zoom-up. Thus the system is capable of processing various stereo data sets with the generic approach and achieves stable photogrammetric accuracy of resultant DTMs. To demonstrate the potential of this stereo processing routine, the DTMs obtained from various Mars orbital images covering some sample test sites were processed with the prototype processor. As the result, the processed DTMs clearly illustrated detailed geological features and high agreement with the height spots of Mars Obiter Laser Altimeter (MOLA). It was proved that the overall processing strategy in this paper was effective and the topographic products were accurate and reliable.
\end{abstract}

Key words: DTM, stereo analysis, Mars, extraterrestrial mapping.

\section{Introduction}

With appropriate imagery and reliable 3D topographic data (e.g. Digital Terrain Model (DTM)), geological mechanisms responsible for the topographic formation in planet surface can be analyzed and interpreted accurately. Therefore, together with the on-site active measurement, optical imagery taken by remote sensing instruments is applied broadly for exploring terrain surfaces of planets.

The worldwide effort devoted to the development of instrumentation and space science technology for the last few decades highly improved the reliability of remote-sensed image of the planetary surface. As the spatial resolution of in-orbital images is updated up to sub-meter level, inorbital planetary images can provide visual observation of detailed terrain features. In addition to the improvement of spatial resolution, the pointing accuracy of in-orbital planetary image integrated from radio tracking and the navigation equipment was also enhanced. Hence, accurate photogrammetric DTM can be produced if stereo images are available. In order to fully benefit from these products, it is essential to develop a processing tool to convert the remote sensing data, especially stereo optical image, to geodetically controlled topographic products. Also, we realized that it is not efficient to establish specific stereo processor for each

Copyright (C) The Society of Geomagnetism and Earth, Planetary and Space Sciences (SGEPSS); The Seismological Society of Japan; The Volcanological Society of Japan; The Geodetic Society of Japan; The Japanese Society for Planetary Sciences; TERRAPUB.

doi:10.5047/eps.2012.11.004 in-orbital planetary optical sensor as has done for last half century in planetary exploration. Hence, we proposed a generic stereo processor employing universal sensor model and pyramidal geodetic control. In theory, it can be employed for any of planetary images within data hierarchy covering the target surface from low resolution to high and very high resolution. To prove the capability and transferability of the system, the pilot scheme employing universal approach with Martian stereo imagery was developed. The processing principles, design of the system and validation are introduced in the following sections. In addition, the potential of prototype system to be utilized for processing stereo data derived from other planetary surfaces was discussed.

\section{Martian Stereo Processing}

Mars is an ideal place to test generic planetary stereo analysis system as pyramidal data hierarchy in in-orbital imagery (from coarse- to high-resolution) is well established. Also, from the user's perspective, the demand of high-resolution topographic datasets for analyses of geological, climatic and potentially exobiological evolution of the Mars has been rapidly increasing. Therefore, the prototype of stereo processing system was tested with Martian imagery in this study.

In order to precisely perform geological analysis and geomorphological interpretation on Mars, the first topographic product was created based on the elevation measured by the Mars Orbiter Laser Altimeter (MOLA, Smith et al., 2001) 
onboard the Mars Global Surveyor (MGS). Due to the characteristics of geodetically verified accuracy and global coverage, the MOLA point set has been treated as the geodetic reference data as well as the base standard for Martian topographic products derived from various Mars exploration missions (Neumann et al., 2001). The MOLA points were further interpolated into regular gridded format with the best resolution of $463 \mathrm{~m}$ per pixel (Jaumann et al., 2007).

For the explorations of Mars surface using remote-sensed imagery, stereo pair taken by MGS Mars Orbiter CameraNarrow Angle (MOC-NA) which possessed 1.5-5 m spatial resolution in mage, was successfully applied for the purposes of geological studies and rover landing site selection (Kirk et al., 2003). Following the MOC-NA system, the High Resolution Stereo Camera (HRSC) carried on board the Mars Express began to provide stereo imagery since January 2004. This nine-look along-track stereo camera system was specially designed to meet the requirements of photogrammetry and cartography for mapping the complete surface of the Mars with the maximum $12.5 \mathrm{~m}$ resolution in nadir mode (Albertz et al., 2005). The acquired image data were then processed to create DTM using DLRVICAR software, in which the MOLA data was adopted for the control of exterior orientation of HRSC stereo imagery (Scholten et al., 2005). With the geodetic control strategy, the vertical accuracy of HRSC topographic products up to 40 m (Spiegel, 2007; Heipke et al., 2007) was achieved. Due to the satisfactory performance of DLR-VICAR, the HRSC topographic products have been systematically created.

In late 2006, the successful deployment of the NASA Mars Reconnaissance Orbiter (MRO) with the High Resolution Imaging Science Experiment (HiRISE) and Context Camera (CTX) instruments began to provide repeatpass stereo image pairs and an opportunity to produce very high resolution DTMs (Malin et al., 2007; McEwen et al., 2007). Compared with HRSC imagery with 12.5 m resolution, very detailed topographic features, such as the gully, dunes, caves and periglacial structures, can be observed in the HiRISE and CTX images whose spatial resolution is $25 \mathrm{~cm}$ and $6 \mathrm{~m}$ respectively. To exploit such high resolution imagery, the Astrogeology Team at the United State Geology Survey (USGS) developed a stereo processor employing Integrated Software for Imagers and Spectrometers (ISIS) (Anderson et al., 2004) and the commercial SOCET SET software (Kirk et al., 2008) to produce HiRISE and CTX DTMs. NASA Ames Research Center also established a planetary stereo processing line (Broxton and Edwards, 2008). Based on the concept applied in the HRSC DTM production line, MOLA data was introduced in these two processing systems to control geodetic quality of the HiRISE and CTX DTMs.

Although the employment of MOLA data in HRSC DTM processing has been successful, Kim et al. (2007) observed that the MOLA data was not able to provide sufficient ground control for verifying HiRISE and CTX DTMs geodetically due to the significant variation of spatial resolution of MOLA DTM, HiRISE and CTX images. To address the issue, Kim and Muller (2009) implemented a "from-coarse-to-fine" workflow to apply MOLA DTM and intermediate HRSC topographic products as the geodetic control for processing HiRISE and CTX images. Furthermore, the geodetic registration of multiple DTMs could be enhanced using surface matching technique developed by Lin et al. (2010). The technical detail of surface matching can be found in Mitchell and Chadwick (1999) and Mills et al. (2003). The DTMs produced through that workflow were verified and played an important role in understanding geological evolution (Warner et al., 2009, 2010a, b, c, 2011).

However, it should be noted that the coverage of very high-resolution data such as HiRISE, was far less than full coverage of Martian surface. Therefore, for researches requiring observation of wide study area, topographic products covering broad extent was necessary. To this end, the paper focused on the extensive planetary topographic mapping over Mars with the hierarchical topographic refinement employing the generic stereo processor. Based on the method reported in Kim and Muller (2009), an advanced hierarchical planetary topography refinement system employing feed-forwarded-model-based matching was developed. The design of the prototype system and detailed workflow were introduced firstly in this paper. Subsequently a number of test sites, including Eberswalde crater, Warrego Valles and Elysium Planitia, were chosen for demonstrating the processing capability of the proposed routines. To show the quality of the resultant DTMs, an assessment was performed through model visualization and comparison with MOLA height spots.

\section{Design of the Processing System}

The system implementation was designed for the generic planetary stereo image processing. The main improvement of this system compared with Kim and Muller (2009)'s original was the feed-forward delivery of DTM product in each processing hierarchy for the model-based matching. Model-based stereo analysis has been widely used for stereo interpolation (Palaniappan et al., 1995). At the stage of image matching, a rough DEM covering area identical to the input stereo images can be used to estimate the disparity through sensor model. As the result, the refinement of the DTM produced from the input stereo can be achieved efficiently. Take Mars data as an example, HRSC DTM can be employed for the initial disparity estimation of CTX stereo.

Due to the imagery data collected from various space missions of Martian surface, a pyramidal hierarchy of topographic data with various spatial resolutions was well established. The global Martian surface is covered by MOLA DTM with $468 \mathrm{~m}$ resolution in equator. Medium resolution stereo data sources from HRSC occupy $50 \%$ of the surface with the improved spatial resolution $(<50 \mathrm{~m}$ per pixel) according to Jaumann et al. (2007). Stereo imagery acquired by CTX and HiRISE cover a very small part of Martian topography however with very high resolution up to $6 \mathrm{~m}$ and sub-meter. As the comprehensive topography is available, the stereo processing using hierarchical refinements between each stereo data sources and MOLA base topography can be established accordingly. The overall workflow for processing Martian remote-sensed data is shown in 


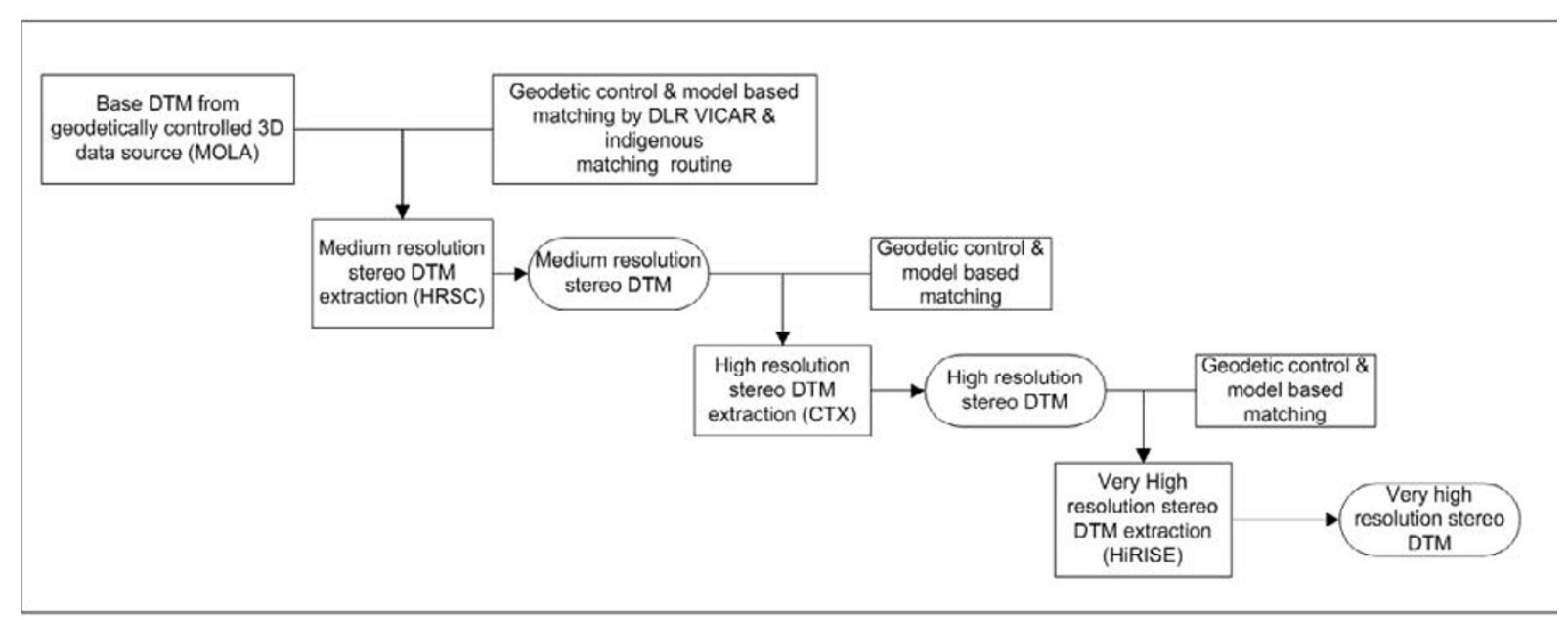

Fig. 1. System configuration for stereo and data fusion processors in Martian surface.

Fig. 1. DTM generated at a processing step was delivered to next stage and then used to estimate the initial disparity in the stereo processors, so called model-based stereo processing. For example, the HRSC DTM was re-employed for the next level CTX stereo processing line to estimate the initial disparity roughly. It was then refined by the image matchers developed based on Adaptive Least Squared Correlation (ALSC) algorithms (Gruen, 1985) which possess sub-pixel matching capability. The search area by the stereo matcher was greatly reduced and the quality of stereo products was able to attain high level consistently. The processing workflow was found highly efficient to process extensive stereo coverage with maximum details. Although the spatial resolution between Martian DEMs was different, it was noted that the proposed model-based matching performed successfully among the data sets. For example, the gridded MOLA DEM with $500 \mathrm{~m}$ spatial resolution for initial disparity estimation of HRSC 12 m nadir image reduced searching area effectively. It was because the disparity range within a grid size of base DEM was constrained to the limited natural slope formed by gravitation.

In addition to the feed-forward model-based matching, another key technique of this strategy was the establishment of unified sensor modeling. With the best photogrammetric quality, accurate photogrammetric co-registration information could be obtained. As the result, the quality of the stereo output was able to fulfill the requirements of scientific researches. Compared with existing planetary stereo processing lines, the distinguished characteristics of this approach were: (1) geodetic accuracy of the resultant topographic products could be verified (refer to Subsection 4.3 for details); (2) the "from-coarse-to-fine" hierarchical processing strategy (HRSC-CTX-HiRISE) for geodetic control was successfully performed to produce Martian DTMs with various resolution; (3) the method could be easily integrated into the high speed processor as the algorithms were generic and could be implemented by spatially divided sub components. To demonstrate the scientific application of this scheme, the test processing over some places of Martian surface was performed and shown in Section 4.

\section{Extensive Martain Topographic Products}

Topographic products covering three testing areas, including Eberswalde Crater, Warrego Valles, and Elysium Planitia, were created using the system shown in Section 3. The geological setting, resultant products and assessment of the testing areas are introduced below.

\subsection{Geological setting of testing areas}

Eberswalde Crater is an impact crater located at $24.2^{\circ} \mathrm{S}$ latitude and $326.5^{\circ} \mathrm{E}$ longitude in the southern highlands of Mars, near north of Holden crater. It is part of the longest Martian lake chain system in Margaritifer Sinus implying the humid climate and the existence of groundwater. The hydrological channel meandering in estuary of Eberswalde Crater was an evidence of fluvial interaction for the deposit of crater. The crater is a drainage basin for a $\sim 90 \mathrm{~km}$-long fluvial network where the channel system debouches into the crater. $\mathrm{A} \sim 150 \mathrm{~m}$-thick fan of material is distributed across $10 \mathrm{~km}$ by $25 \mathrm{~km}$ of the crater floor (Malin and Edgett, 2003). The formation of deposit within crater and the conditions of the formation are still under discussion. Malin and Edgett interpreted it as an exhumed and inverted remnant of fluvial distributary network in late Noachian age. The possible crater lake proposed by fluvial-lacustrine model suggested that the Martian surface condition had been sustained a large amount of liquid water in stable state so that the deposits were able to form a significant delta structure. Moore et al. (2003) estimated persistent $700 \mathrm{~m}^{3} / \mathrm{sec}$ water based on the channel width and meander wavelength. If the origin of water flow was the quasi-periodic climate episodes involving typical terrestrial-style precipitation, it required $10^{3}-10^{6}$ years to form the whole deposit according to the assumption. A quantitative analysis of the exposed stratigraphy by Lewis and Aharonson (2006) indicated that the delta likely formed not in a stable long-lived lake but over the course of a small number of shorter lacustrine episodes, which were not sustained at equilibrium conditions. Therefore both works supported that the Eberswalde deposit was formed under the episodic humid climate conditions. Pondrelli et al. (2008) observed the topset-foreset-bottomset geometry, which is the typical delta progradation on the Earth, using MOC-NA image superimposed HRSC DTM. Based 
on this evidence, they interpreted the Eberswalde feature as a fan-delta structure.

Warrego Valles is a channel located in Thaumasia quadrangle (center latitude and longitude of this area is $42^{\circ} \mathrm{S}$ and $270^{\circ} \mathrm{E}$ respectively) and across $188 \mathrm{~km}$ in the highland. Judging from the network of branching valleys, its fluvial origin is quite clear. Gulick $(1998,2001)$ firstly noted the similarity with hydrological channel alongside of Hawaiian Kohala volcanoes but valleys have not formed all along the plateau boundary as might be expected from rainfall. The proof of hydrothermal and snow melting activities in this area might exist. Ansan and Mangold (2006) proposed the possibility of precipitation and surface runoff to cut off the fluvial channel in this area using THEMIS and MRO-NA images. Rossi et al. (2008) proposed the existence of periglacial landforms especially rock glacier. Recent HiRISE images showed complicated textures which may be evidence of the sublimation proposed by Mangold (2003). Therefore it is now very clear there was once a large amount of ice and molten water in the valley networks. The newly established CTX and HiRISE DTMs could provide powerful tools to model fluvial activities in Warrego Valles. For example, the fine resolution stereo DTM could give a clue of the extents of rock glacier activities and the amount of sublimated ice. To this end, the high-resolution stereo DTMs were updated in the proposed system.

The Elysium Planitia (center latitude and longitude of this area is $2^{\circ} \mathrm{N}$ and $155^{\circ} \mathrm{E}$ respectively) is a controversial area due to a complex history of recent fluvial (Burr et al., 2002, 2005; Murray et al., 2005) and volcanic (Plescia, 2003; Jaeger et al., 2007) activities. The central valles over Elysium Planitia called Athabasca were likely formed by catastrophic flood, which was probably originated from the groundwater eruption by the thermal activities in Cerberus Fossae (Head et al., 2003). This event was dated as a very recent geological activity. Head et al. (2003) proposed a model of the magmatic dike emplacement involving attendant cryospheric cracking to fracture the surface and release pressurized groundwater confined beneath the cryosphere. Then the water effused along a segment of the fracture to form Athabasca Valles and was flowed into Elysium Planitia. The estimated water flow through a dikerelated cryospheric fracture was $\sim 2 \mathrm{~m}$ wide, at water rise speeds of $\sim 60 \mathrm{~m} / \mathrm{s}$. These values were well suited with the total discharge estimation of $10^{6} \mathrm{~m}^{3} / \mathrm{s}$ over Athabasca Valles and MarteVall is reported by Burr et al. (2002). In addition, the teardrop islands and the sediment over bedrock in the Athabasca Valles were very supportive evidences for the recent fluvial in the central channel. In 2005, HRSC images revealed geomorphic features described as ash covered packed ice (Murray et al., 2005). Therefore, it was inferred that Burr et al.'s interpretation (2002) was well suited with the newly discovered packed ice model. On the contrary, Jaeger et al. (2007) argued that polygonal, ridged flow textures and widely populated cone structures (so called ring-mound landforms) observed in detail by HiRISE image were the results of thin low-viscosity fluid flow from the Cerberus Fossae-so thin, solid, and rafting crust of an active lava flow. Later, Jaeger et al. (2010) proposed a single eruption covering $250,000 \mathrm{~km}^{2}$ of western Elysium
Planitia with an estimated $5,000-7,500 \mathrm{~km}^{3}$ of mafic or ultramafic flood lava emplaced turbulently over a period of several weeks. In consequences, the flood lava replaced the aqueous floods in the magnitude and dynamics to incise the channels according to their propositions. As summarized above, it was realized that the arguments about the formation mechanisms of Athabasca Valles and Elysium Planitia are still undergoing. To understand the geological implication over Elysium Planitia, the multi-resolution DTMs would provide crucial information, such as the finest terrain over the floor of valles and the tear-drop shaped island structures.

\subsection{Processing and results of topographic products}

The processing of stereo DTMs followed the workflow described in Section 3. The topographic products were constructed and refined in the order of MOLA, HRSC, CTX and HiRISE data. Firstly, MOLA DTM over the testing area was delivered into the DLR-VICAR for producing HRSC Level 3 ortho-image. Stereo matching was performed in the Level 3 image plane and the matched points in the stereo were back-projected into Level 2 image plane where the space intersection was executed based on HRSC sensor model (refer to Scholten et al. (2005) for detailed HRSC processing workflow). In order to obtain sufficient and correct matching point at this image matching stage, the two-stage matcher consisting of the front-end part and the ALSC refinement (Kim and Muller, 2009) was applied. Once the first HRSC DTM was produced, it was fed forward to re-generate the HRSC Level 3 ortho-image and then run the two-stage matching again. The procedure was performed iteratively. It was found that the matching blunders were largely reduced and the details of topography were significantly enhanced through the proposed image matching strategy. Regarding the processing of CTX stereo pairs over major geological features, a number of ground control points (GCPs, typically $>10$ points) for the update of sensor model, were selected around gentle slope areas manually. It should be noted the semi-automated GCP selection method was already developed and applied for the stereo processing. However, as GCP setting over high relief area or the unbalanced GCP distribution implemented by semi/full-automated GCP selection might result in instability of sensor model occasionally, the GCP selection was performed manually in this paper in order to achieve an accurate geodetic control. For image matching of CTX stereo pair, the model-based two stage matching method was applied again, of which the resultant HRSC DTM was fed forwarded to be used for ortho-rectifying the CTX images. Subsequently the iterative image matching scheme demonstrated in HRSC processing was performed to generate CTX DTM. HiRISE stereo DTM was also produced following the processing scheme of CTX data.

In addition to the iterative model-based matching, the other key factor of such hierarchical processing was the accuracy of the sensor models. Since the strategy in this study was working under the condition that all established sensor models were co-aligned, the successful "from-coarse-tofine" geodetic control proposed by Kim and Muller (2009) was the prerequisite for the high quality products. Once the generic sensor model is successfully controlled, the stereo 
(a)

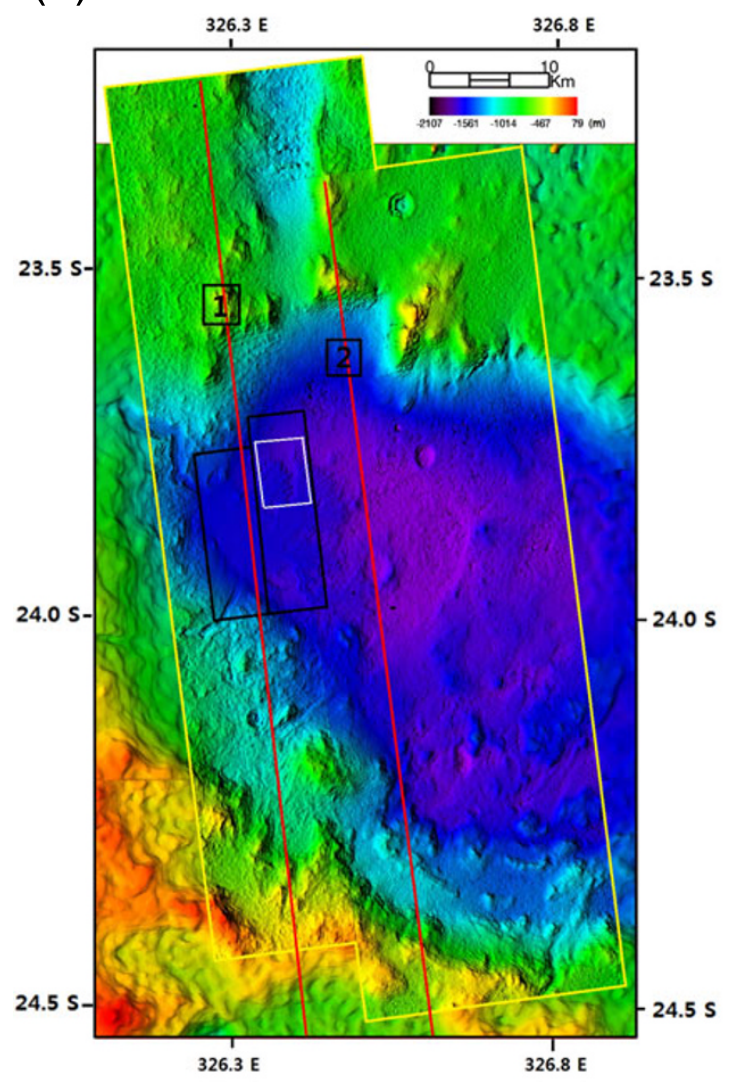

(c)

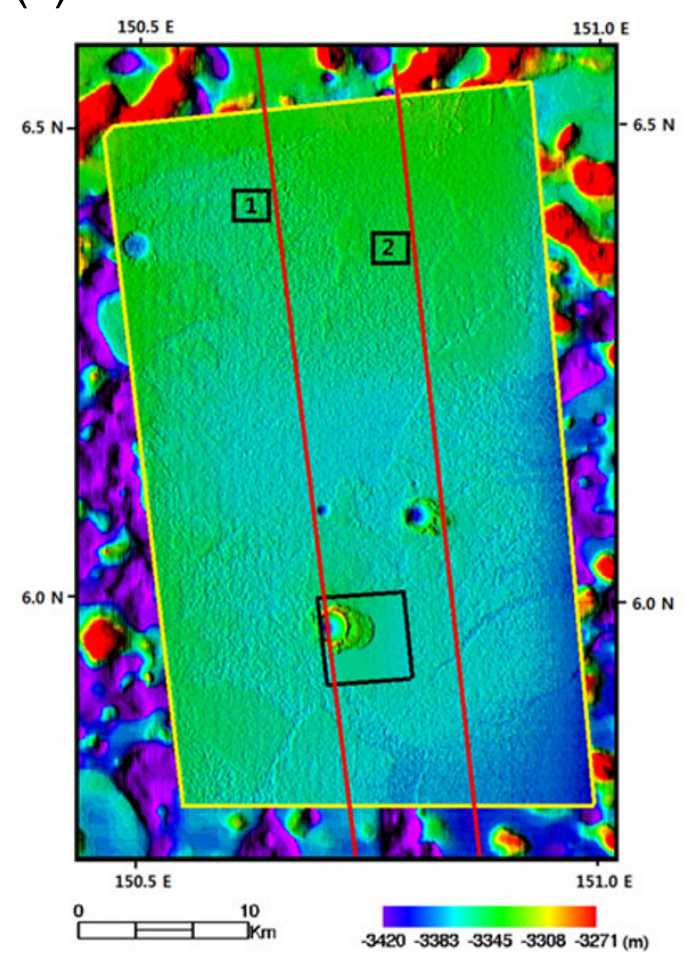

(b)

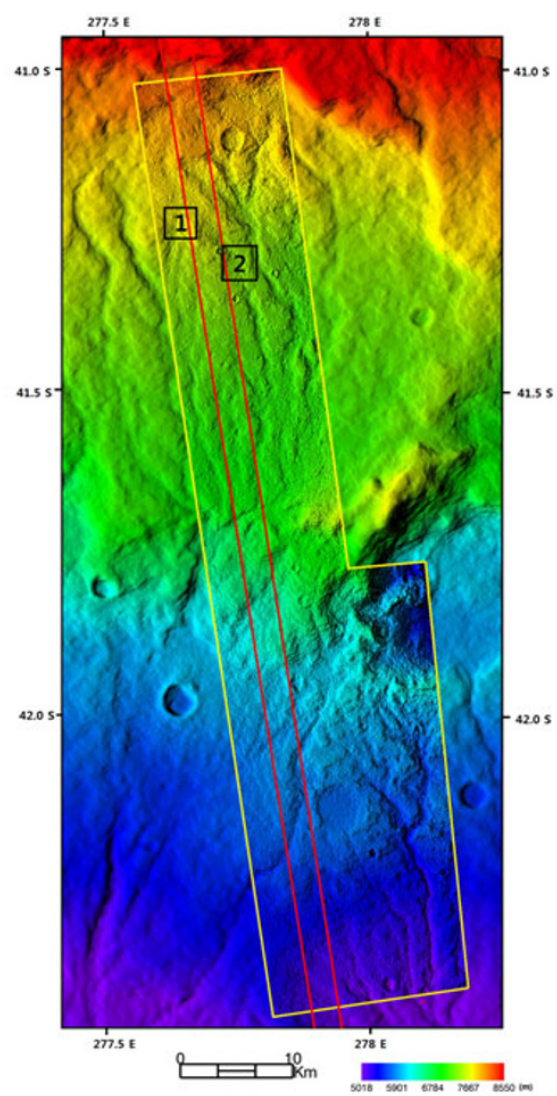


(a)
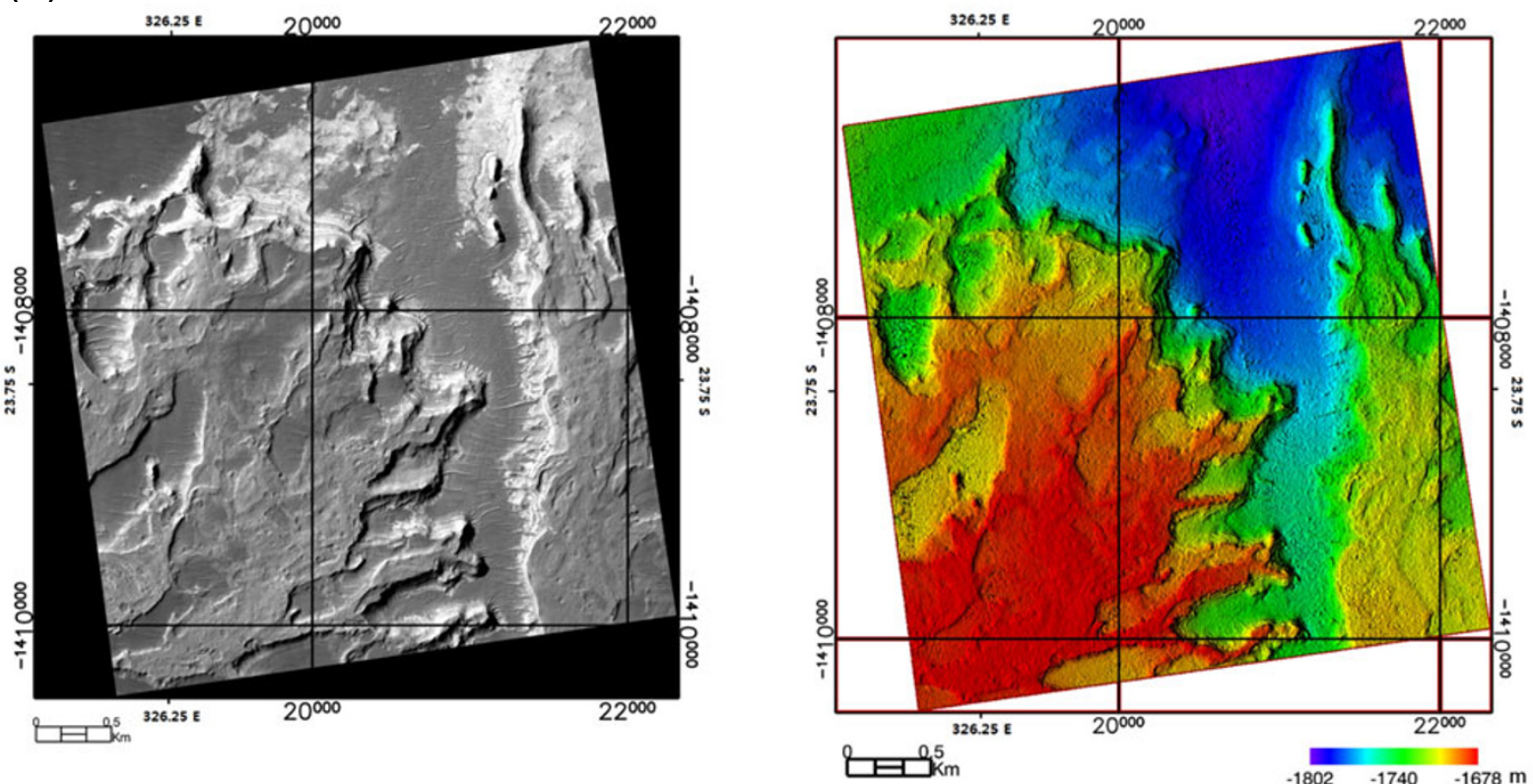

(b)
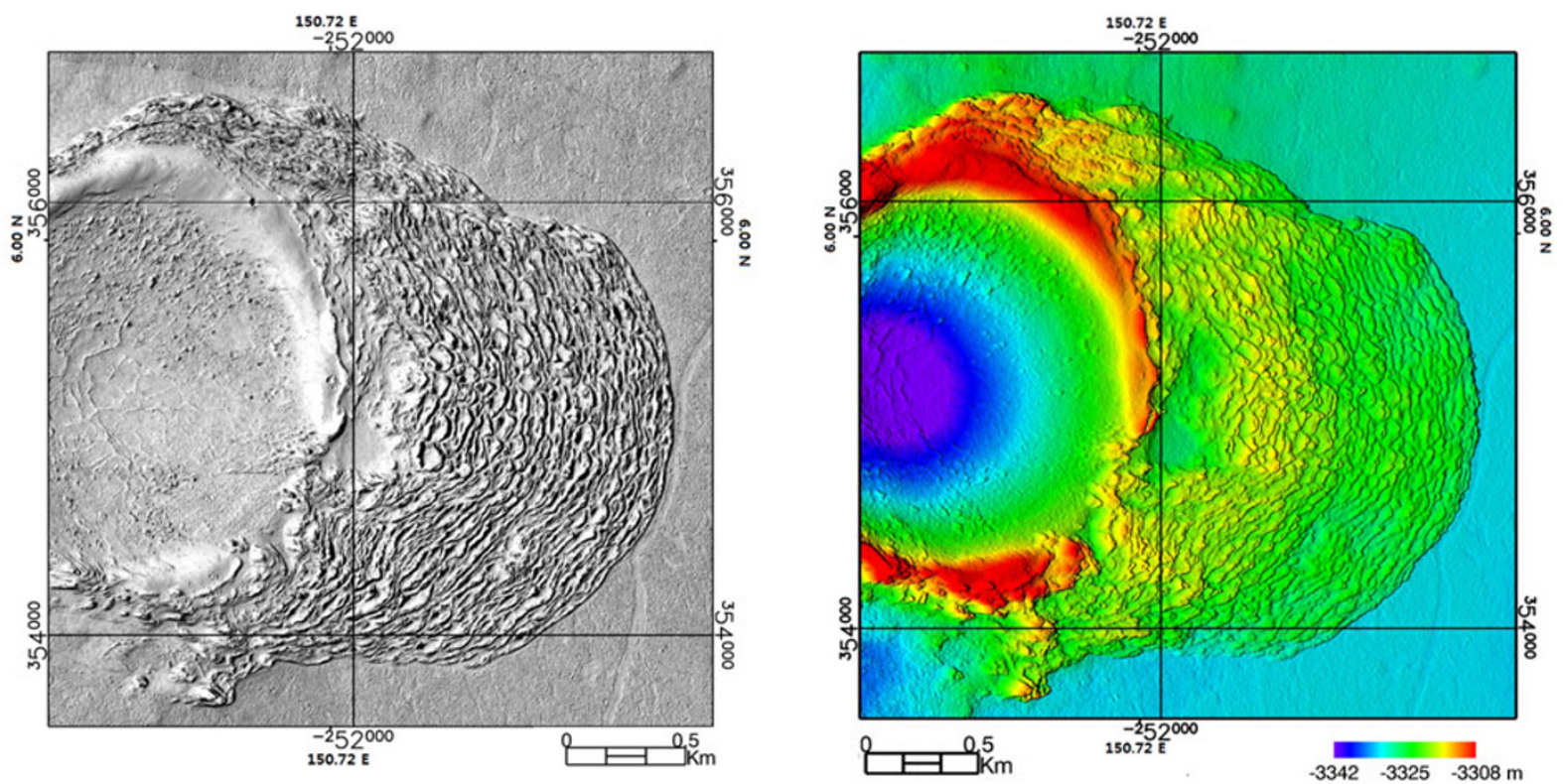

Fig. 3. Stereo DTM products extracted from HiRISE with maximum possible resolution High resolution ortho image (left, $0.4 \mathrm{~m}$ resolution) and stereo DTM ( $1 \mathrm{~m}$ grid spacing) over left flank of Ebersewalde deposits. Sinusoidal coordinate system with 326.0 central longitude was used. High resolution ortho image (left, $0.4 \mathrm{~m}$ resolution) and stereo DTM (0.7 m grid spacing) over Elysium Planitia. Sinusoidal coordinate system with 150.0 central longitude was used.

products were accurately co-aligned within a 3D surface plane and the seamless DTM product over whole target area was accomplished. Under such circumstance, the hierarchical refinements were effectively performed in the places where the stereo pair was available.

Extensive DTMs and sub-meter grid spacing DTM of specific areas produced using this method are shown in Figs. 2 and 3 respectively. Extensive DTM covering Eberswalde Crater was produced based on one HRSC (h2013_0000), two CTX (P01_001336_1560, P01_001534_1559 and B02_010474_1558, P01_010553_1558) and two HiRISE stereo pairs (PSP_001336_1560, PSP_001534_1560 and PSP_001336_1560, PSP_001534_1560) as shown in Fig. 2(a). As the area had been proposed as the candidate of the rover landing site, comprehensive collection of multiple stereo pairs was available. From the resultant fused DTM of Eberswalde Crater, the seamless mosaicked DTM was observed. This was the consequence of the extremely correct geodetic control. Due to this advantage, the route planning of the rover could be conducted on the extensive DTM. Furthermore, the main lobe of carter deposit shown in Figs. 2(a) and 3(a) could be measured three dimensionally with refined $1 \mathrm{~m}$ resolution HiRISE DTM to trace the 
epoch of fluvial sediment deposit. Overall, it is realized that the quality of the DTM played a key role for correctly determining fan deposits and morphologic parameters of the channel network system. Compared with the MOC-NA stereo pair employed in Moore et al. (2003) and Lewis and Aharonson (2006), the DTMs reconstructed from high-resolution HiRISE and CTX stereo imagery definitely improved the correctness and efficiency for geological and morphologic analyses.

Stereo DTMs of Warrego Valles and Elysium Planitia are demonstrated in Figs. 2(b), 2(c) and 3(b). Compared with the Eberswalde Crater, the stereo geodetic controlling was performed under harsh conditions. Over Warrego Valles, the target CTX stereo pair (P07_003633_1378 and P08_004266_1377) was taken under long exposing time (23 times than the normal CTX image strip) of CCD over steep slope terrain. As the sensor to target relationship cannot be simply interpolated into generic function, the accurate sensor modeling especially in the regression of pointing information was therefore difficult to achieve. Note that basically the sensor model was the mapping function from target to image coordinate. The employment of the high order polynomial form to address in the generic sensor model would increase the instability. Consequently, the constructed stereo terrain was slanted into one direction and the discontinuities distributed along the boundary of CTX and HRSC DTMs (h0453_0000) were obvious. To address this issue, the non-rigorous sensor model was built up only in a portion of CTX pairs and applied in stereo interpretation. However, the discontinuity up to $60 \mathrm{~m}$ vertically still remained along upper left portion of CTX DTM as shown in Fig. 2(b). The details in fluvial channels and crater could be clearly observed and the dimension was measureable with the stereo DTM. As for the HiRISE data of Warrego Valles, mismatching CCD overlapping flaw was found in the acquired stereo pair. This was caused by the high frequency oscillation of spacecraft (McEwen et al., 2007) and the proper solution using USGS's ISIS software was proposed by Kirk et al. (2008). Together with the CCD mismatching issue, complicated geological features extensively populating over Warrego Valles also affect the performance of tie points setting algorithm between CCD lines in USGS ISIS. Due to the factors described above, the processing of HiRISE stereo pair in Warrego Valles will be possible after the publication of USGS HiJACK routine (Mattson et al., 2009), with which the interruption in CCD boundaries was minimized. With the resultant high-resolution 3D topography of HIRISE, it is expected that the transportation mechanism interpretation can be applied to identify the origin of Warrego Valles.

The geological events occurring in Elysium Planitia, probably the fluvial activity (Burr et al., 2002) or the flood lava eruption (Jaeger et al., 2010), created a very flat surface on Mars. The maximum height difference over the hundreds kilometer squared target areas was less than $30 \mathrm{~m}$. Due to the flat terrain surface, the stereo image matching as well as the geodic control became challenge tasks in the processor, because both procedures depended on the identification of the corresponding features and the textures between images. As a result, any insignificant ver- tical errors of ground control points determined in HRSC base DTM from h2165_0000 would result in overall tilting in the CTX stereo (G01_18537_1861 and G0219170_1861) topography. Therefore, the surface matching tool (Lin et al., 2010) was applied to improve the quality of geodetic control between MOLA and stereo CTX DTM. Once the matching was convergence, the tilting in CTX DTM was successfully addressed. This is observed through the detailed measurement of the geodetic quality improvement and the comparison with MOLA spots (refer to Subsection 4.3). Such difficulty in geodetic control was not found in the HiRISE DTM processing procedure, as the HiRISE stereo (ESP_18537_1860 and ESP_019170_1860) is located in the middle of CTX coverage where the tilting effect is minimum. Consequently, the resultant HiRISE DTM was well established with stable geodetic accuracy (Fig. 3(b)).

From the description of the workflow and DTM products demonstrated in Figs. 2 and 3, it was realized that no manual DTM editing was necessary and obvious matching blunders were not observed in the final DTM. The reliability and effectiveness of the model-based matching strategy exploiting data hierarchy was proved. Figure 3(a) and 3(b) demonstrated a DTM products created with the maximum grid spacing in test areas with HiRISE. The maximum HiRISE DTM grid spacing depends on the stereo image quality but normally $0.5-0.7 \mathrm{~m}$ was achievable with the developed matching scheme. Clearly the hierarchical approach for the model-based matching in the stereo implementation is capable of producing sub-meter topography with HiRISE image pair.

\subsection{Geodetic control assessment}

The quality assessments of the products employing MOLA height points showed the highly accurate geodetic controls over the stereo DTM coverage in Fig. 4. Eberswalde showed extremely good agreement between stereo DTM and MOLA height spot as shown in Fig. 4(a). The discrepancies as observed in the side portions of Fig. 4(a) may result from some matching blunder in the steep slope topography and partly not fully addressed photogrammetric control. However, overall geodetic accuracy is highly accurate even compared with the terrestrial stereo products made by the commercial satellites which have normally decameter scale shifts in horizontal and vertical direction (Kim and Muller, 2007). A little discrepancy between MOLA and stereo topography in Fig. 4(b) over Warrego shows a possibility of small horizontal and/or vertical shift of constructed DTM originated from the long expositing time of stereo images. Even though the validation result in Fig. 4(c) over Elysium Planitia revealed the possibility of tilting of CTX DTMs as explained in Subsection 3.2, the improved DTM applying the surface matching with MOLA spots showed fine agreement. It was implied that the surface matching with the reference data sets could be a solution in some specific stereo coverage where the geodetic control point setting was not reliable due to the absence of significant feature texture.

\section{Discussion}

Topographic products generated through the processing scheme proved the effectiveness of the main idea employing 

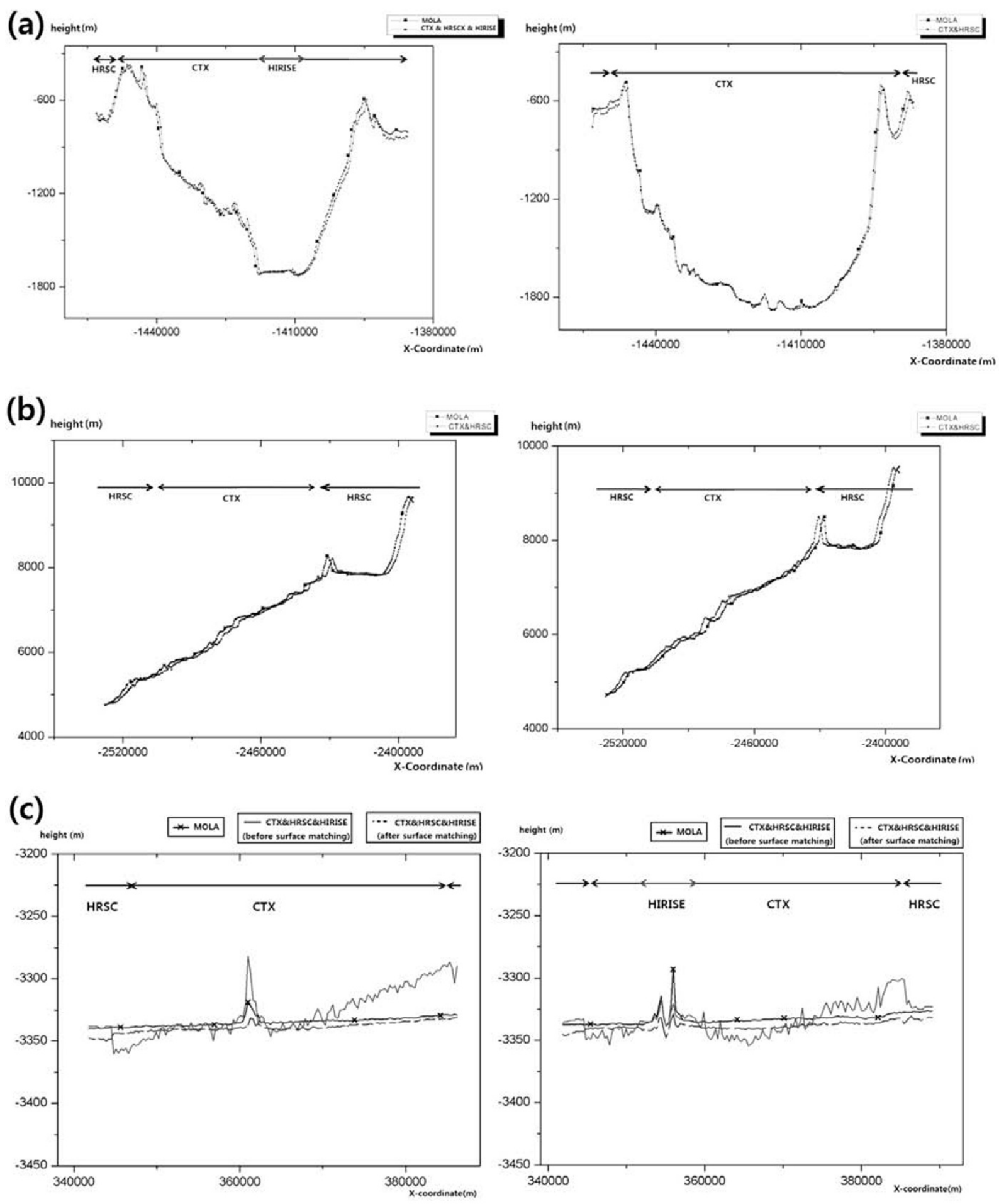

Fig. 4. Stereo DTM assessments compared with MOLA spots. The upper arrowed lines used for the assignment of employed DTMs for the profiling. Note that the red arrowed line indicates the HiRISE DTM used for the comparison. (a) MOLA track profiling with stereo DTM over Eberswalde cater (mean value of MOLA-CTX height difference: $9.9 \mathrm{~m}$, standard deviation (Std. Dev.) of height difference: $28.8 \mathrm{~m}$ ) left for track 1 and right for track 2 respectively. (b) MOLA track profiling with stereo DTM over Warrego Valles (mean value of MOLA-CTX height difference: $15.4 \mathrm{~m}$, Std. Dev. of height difference: $32.8 \mathrm{~m}$ ) left for track 1 and right for track 2 respectively. (c) MOLA track profiling with stereo DTM over Elysium Planitia (mean value of MOLA-CTX height difference: $3.2 \mathrm{~m}$, Std. Dev. of height difference: $5.5 \mathrm{~m}$ after surface matching, mean value of MOLA-CTX height difference: $5.4 \mathrm{~m}$, Std. Dev. of height difference: $10.2 \mathrm{~m}$ before surface matching), left for track 1 and right for track 2 respectively.

stereo data hierarchy. Since the geodetic control was well performed by from-coarse-to-fine approach, the modelbased stereo processing was able to produce highly reliable topographic products. As these datasets are obtained from different sensors and have various characteristics, there will be additional benefits if multiple topographic products can be analyzed and/or exploited simultaneously. For example, as the CTX DTM and ortho-image can be adjusted to make 
seamless mosaic with HRSC topographic data, CTX stereo products provided a great opportunity for scientists to observe 3D topography over more extensive area. In addition, the finest processing with HiRISE data produced even submeter level topographic products in which unprecedented detailed geomorphic characteristics were shown, and the highly accurate quantitative geological interpretation can be performed accordingly.

Since the production of Martian stereo topographic products was proved successful, the system developed in this study is further proposed as the prototype system capable of processing planetary data possessing the same kind of data hierarchy. For example, lunar surface covered by Lunar Orbiter Laser Altimeter (LOLA), image data acquired by Lunar Reconnaissance orbiter (LRO) Wide Angle (WA) and Narrow Angle (NA) cameras can be processed with such processing scheme. Also, Mercury with Mercury Laser Altimeter (MLA) and optical imagery of Messenger can be the candidate for the application of proposed stereo system. Based on the main components have been accomplished in the developed system, including geodetic control, the scheme of stereo image matching and the feed-forwarded data delivery for model-based matching, the prototype system can be executed with a few additional modules for accommodating specific characteristics of applied data.

It is found that extremely high spatial resolution and blunder-free topographic products are gradually required for supporting broad scientific topics, such as small scale geomorphic interpretation, landing risk assessment and route planning for planetary exploration rover. To fulfill the requirements, topographic products created from the current processing scheme can be further updated. This task can be tackled with an approach combining the matching blunder detection scheme and the application of shapefrom-shading technique. This approach can be straightforwardly integrated into the existing system to improve the quality of stereo products by suppressing blunders through the comparison procedure. Meanwhile, in order to handle the increased computing cost comes with the designed processing modules and huge image size, Graphics Processing Unit (GPU) and/or parallel processing which is readily to be incorporated with the "from-coarse-to-fine" geodetic control and the unified sensor models are essential for the all capabilities described above. With the updated processing modules and advanced computing facility, not only the DTM quality can be improved, but also the implementation of the updated system is of great potential to be performed with high level of fully automated processing of planetary topographic data.

Final discussion goes to the additional value of the system. Once the overall system is established, the expansion of such stereo analysis tool can be readily implemented over the surface of planets or planets' satellites which are covered by imagery acquired by optical sensors equipped in the future planetary missions. As the non-rigorous sensor model and the geodetic control method are generic, the stereo analysis can start to work once the conversion from original positioning data set (so called Spacecraft-PlanetInstrument-C-matrix for pointing-Event (SPICE), Navigation and Ancillary Information Facility, 2011) to non- rigorous sensor model is achieved.

\section{Conclusions and Future Works}

The deficiency of standard stereo processing system using planetary imagery has been a significant barrier for the geological and geomorphic researches over solid solar system bodies. The primary outcome of this study is the prototype of unprecedented flexible and powerful stereo routines covering a variety of planetary imagery. The stereo processing algorithms employing the non-rigorous sensor model and the "from-coarse-to-fine" geodetic control originally developed by Kim and Muller (2009) were updated to effectively exploit stereo image hierarchy. The system proved its potential by producing DTMs over three testing sites on Martian surface. Due to the high accuracy geodetic control, the model-based stereo matching was successfully produced high-resolution CTX and HiRISE DTMs possessing high geodetic accuracies as well as the blunder free topographies. Also, the HiRISE stereo DTMs showed that the maximum possible DTM resolution is up to sub-meter level.

Since the planetary topographic data with medium and very high spatial resolutions provide scientific researchers a unique chance to observe and interpret three-dimensional surface, many research works involving geological analysis will get benefits from the outputs of the processing scheme in this study. However, as discussed in Section 5, the current stereo scheme for planetary data employing conventional computing technology will be soon meet difficulties to manipulate the huge number and size of future planetary images, such as images acquired by High-resolution Stereo Color Imager (HiSCI, McEwen et al., 2011). For example, one pair of HiRISE stereo processing requires a billion of matching operations. Additionally, if higher accuracy is requested, more computer operations are further required to refine the accuracy into sub-pixel level. With conventional approach, the computing cost to build a DTM scene with the contemporary and future sensor is definitely not enough. It should be noted that the full utilization of the stereo routines requires huge computing power which is achievable by the application of advanced GPU/parallel computing. Due to the application of unified sensor model and stereo matching component, the approaches proposed in this paper is highly suitable for the construction of parallel/GPU processing line.

Once such conditions are satisfied, the proposed system becomes a powerful tool to extract topographic data with advanced efficiency, highly precise geodetic control and minimum manual interaction. Together with the system improvement, it should be noted that the system is capable of processing stereo data of other solid body planetary, such as Mercury and Moon with minor modification.

Acknowledgments. The first author thanks to Ms. HeWon Yun for her efforts in model visualizations. We are grateful to Professor Sanjeev Gupta and Dr. Nick Werner for their helpful comments.

\section{References}

Albertz, J., M. Attwenger, J. Barrett, S. Casley, P. Dorninger, E. Dorrer, H. Ebner, S. Gehrke, B. Giese, K. Gwinner, C. Heipke, E. HowingtonKraus, P. L. Kirk, H. Lehmann, H. Mayer, J.-P. Muller, J. Oberst, A. 
Ostrovskiy, J. Renter, S. Reznik, R. Schmidt, F. Scholten, M. Spiegel, U. Stilla, M. Wahlisch, G. Neukum, and the HRSC CoI-Team, HRSC on Mars Express-Photogrammetric and cartographic research, Photogramm. Eng. Rem. S., 71(10), 1153-1166, 2005.

Anderson, J. A., S. C. Sides, D. L. Soltesz, T. L. Sucharski, and K. J. Becker, Modernization of the Integrated Software for Imagers and Spectrometers. The 35th Lunar Planet. Sci., Abstract \#2039, 2004.

Ansan, V. and N. Mangold, New observations of Warrego Valles, Mars: Evidence for precipitation and surface runoff, Planet. Space Sci., 54, 219-242, 2006.

Broxton, M. J. and L. J. Edwards, The Ames Stereo Pipeline: Automated 3D Surface Reconstruction from Orbital Imagery, The 39th Lunar Planet. Sci., Abstract \#2419, 2008.

Burr, D. M., J. A. Grier, A. S. McEwen, and L. P. Keszthelyi, Repeated aqueous flooding from the Cerberus Fossae: Evidence for very recently extant, deep groundwater on Mars, Icarus, 159(1), 53-73, 2002.

Burr, D. M., R. J. Soare, J. W. B. Tseung, and J. P. Emery, Young (late Amazonian), near-surface, ground ice features near the equator, Athabasca Valles, Mars, Icarus, 178, 56-73, 2005.

Gruen, A., Adaptive least squares correlation: A powerful image matching technique, South Africa J. Photogramm. Rem. S. Carto., 14(3), 175-187, 1985.

Gulick, V. C., Magmatic intrusions \& a hydrothermal origin for fluvial valleys on Mars, J. Geophys. Res., 103(19), 365-19, 1998.

Gulick, V. C., Origin of the valley networks on Mars: A hydrological perspective, Geomorphology, 37, 241-268, 2001.

Head, J. H., L. Wilson, and K. L. Mitchell, Generation of recent massive water floods at Cerberus Fossae, Mars bydike emplacement, cryospheric cracking, and confined aquifergroundwaterrelease, Geophys. Res. Lett., 30(11), 1577, doi:10.1029/2003GL017135, 2003.

Heipke, C., J. Oberst, J. Albertz, M. Attwenger, P. Dorninger, E. Dorrer, M. Ewe, S. Gehrke, K. Gwinner, H. Hirschmüller, J. R. Kim, R. L. Kirk, H. Mayer, J.-P. Muller, R. Rengarajan, M. Rentsch, R. Schmidt, F. Scholten, J. Shan, M. Spiegel, M. Wählisch, G. Neukum, and the HRSC CoI team, Evaluating planetary digital terrain modelsThe HRSC DTM test, Planet. Space Sci., 55, 2173-2191, 2007.

Jaeger, W., L. P. Keszthelyi, A. S. McEwen, C. M. Dundas, and P. S. Russell, Athabasca Valles, Mars: A lava-draped channel system, Science, 317, 1709-1711, 2007.

Jaeger, W. L., L. P. Keszthelyi, J. A. Skinner, M. P. Milazzo, A. S. McEwen, T. N. Titus, M. R. Rosiek, D. M. Galuszka, E. HowingtonKraus, and R. L. Kirk, HiRISE Team, Emplacement of the youngest flood lava on Mars: A short, turbulent story, Icarus, 205, 230-243, 2010.

Jaumann, R., G. Neukum, T. Behnke, T. C. Duxbury, K. Eichentopf, J. Flohrer, S. V. Gasselt, B. Giese, K. Gwinner, E. Hauber, H. Hoffmann, A. Hoffmeister, U. Kohler, K.-D. Matz, T. B. McCord, V. Mertens, J. Oberst, R. Pischel, D. Reiss, E. Ress, T. Roatsch, P. Saiger, F. Scholten, G. Schwarz, K. Stephan, M. Wahlisch, and the HRSC Co-Investigator Team, The high-resolution stereo camera (HRSC) experiment on Mars Express: Instrument aspects and experiment conduct frominterplanetary cruise through the nominal mission, Planet. Space Sci., 55, 928-952, 2007.

Kim, J. R. and J.-P. Muller, A landcover classification scheme for high resolution imagery and it's application to bushfire protection in residential areas, Isprs Hannover Workshop 2007 High-Resolution Earth Imaging For Geospatial Information, http://www.isprs.org/proceedings/XXXVI/1W51/paper/Kim_muller.pdf, (assessed at 20th Jan 2012), 2007.

Kim, J. R. and J.-P. Muller, Multi-resolution topographic data extraction from Martian stereo imagery, Planet. Space Sci., 57(15), 2095-2112, 2009.

Kim, J. R., J.-P. Muller, M. Balme, and J. B. Murray, Geometric ground control of very high resolution imagery using HRSC intersection points and a non-Rigorous camera model, The 38th Lunar Planet. Sci., Abstract \#1811, 2007.

Kirk, R. L., E. Howington-Kraus, B. R. Redding, D. Galuszka, T. M. Hare, B. A. Archinal, L. A. Soderblom, and J. M. Barrett, High resolution topomapping of candidate MER landing sites with Mars Orbiter Camera narrow-angle images, J. Geophys. Res., 108(E12), 8088, doi:10.1029/2003JE002131, 2003.

Kirk, R. L., E. Howington-Kraus, M. R. Rosiek, J. A. Anderson, B. A. Archinal, K. J. Becker, D. A. Cook, D. M. Galuszka, P. E. Geissler, T. M. Hare, I. M. Holmberg, L. P. Keszthelyi, B. L. Redding, A. W. Delamere, D. Gallagher, J. D. Chapel, E. M. Eliason, R. King, A. S. McEwen, and the HiRISE Team, Ultrahigh resolution topographic mapping of Mars with MRO HiRISE stereo images: Meter-scale slopes of candidate Phoenix landing sites, J. Geophys. Res., 113, E00A24, doi:10.1029/2007JE0030000, 2008.

Lewis, K. W. and O. Aharonson, Stratigraphic analysis of the distributary fan in Eberswalde crater using stereo imagery, J. Geophys. Res., 111, E06001, doi:10.1029/2005JE002558, 2006.

Lin, S.-Y., J.-P. Muller, J. P. Mills, and P. E. Miller, An assessment of surface matching for the automated co-registration of MOLA, HRSC and HiRISE DTMs, Earth Planet. Sci. Lett., 294(3-4), 520-533, 2010.

Malin, M. C. and K. S. Edgett, Evidence for persistent flow and aqueous sedimentation on early Mars, Science, 302, 1931-1934, doi:10.1126/science.1090544, 2003.

Malin, M. C., J. F. Bell, B. A. Cantor, M. A. Caplinger, W. M. Calvin, R. T. Clancy, K. S. Edgett, L. Edwards, R. M. Haberle, P. B. James, S. W. Lee, M. A. Ravine, P. C. Thomas, and M. J. Wolff, Context camera investigation on board the Mars reconnaissance orbiter, J. Geophys. Res., 112, E05S04, doi:10.1029/2006JE002808, 2007.

Mangold, N., Geomorphic analysis of lobate debris aprons on Mars at Mars Orbiter Camera scale: evidence for ice sublimation initiated by fractures, J. Geophys. Res., 108, 8021, 2003.

Mattson, S., A. Byod, R. L. Kirk, D. A. Cook, and E. HowingtonKraus, HiJACK:correcting space craft jitter in HiRISE images of Mars, Euro. Planet. Sci. Congress., http://meetings.copernicus.org/epsc2009 (assessed at 20th Jan 2012), 2009.

McEwen, A. S., E. M. Eliason, J. W. Bergstrom, N. T. Bridges, C. J. Hansen, W. A. Delamere, J. A. Grant, V. C. Gulick, K. E. Herkenhoff, L. Keszthelyi, R. L. Kirk, M. T. Mellon, S. W. Squyres, N. Thomas, and C. M. Weitz, Mars reconnaissance orbiter's High Resolution Imaging Science Experiment (HiRISE), J. Geophys. Res., 112, E05S02, doi:10.1029/2005JE002605, 2007

McEwen, A. S., N. Thomas, J. Bridges, S. Byrne, G. Cremonese, W. Delamere, C. Hansen, E. Hauber, L. Ivanov, N. Mangold, W. J. Markiewicz, M. Massironi, M. Mattson, C. Okubo, and J. Wray, HiSCI Experiment on ExoMars Trace Gas Orbiter, The 42th Lunar Planet. Sci., Abstract \#2270, 2011.

Mills, J. P., S. J. Buckley, and H. L. Mitchell, Synergistic fusion of GPS and photogrammetrically generated elevation models, Photogramm. Eng. Rem. S., 69(4), 341-349, 2003.

Mitchell, H. L. and R. G. Chadwick, Digital photogrammetric concepts applied to surface deformation studies, Geomatica, 53(4), 405-414, 1999.

Moore, J. M., A. D. Howard, W. E. Dietrich, and P. M. Schenk, Martian layered fluvial deposits: Implications for Noachian climate, scenarios, Geophys. Res. Lett., 30(24), 2292, doi:10.1029/2003GL019002, 2003.

Murray, J., J. P. Muller, G. Neukum, S. C. Werner, S. van Gasselt, E. Hauber, W. J. Markiewicz, J. W. Head, B. H. Foing, D. Page, K. L. Mitchell, and G. Portyankina, HRSC Co-Investigator Team, Evidence from the Mars express high resolution stereo camera for a frozen sea close to Mars' equator, Nature, 434, 352-356, 2005.

Navigation and Ancillary Information Facility, http://naif.jpl.nasa.gov/naif/ (assessed at 20th Jan 2012), 2011.

Neumann, G. A., D. D. Rowlands, F. G. Lemoine, D. E. Smith, and M T. Zuber, Crossover analysis of Mars Orbiter Laser Altimeter data, $J$. Geophys. Res., 106(E10), 23753-23768, 2001.

Palaniappan, K., Y. Huang, X. Zhuang, and A. F. Hasler, Robust stereo analysis, Proceedings of International Symposium on Computer Vision, Florida, U.S.A., 175-181, 1995.

Plescia, J. B., Cerberus Fossae, Elysium, Mars: A source for lava and water, Icarus, 164, 79-95, 2003.

Pondrelli, M., A. P. Rossi, L. Marinangeli, E. Hauber, K. Gwinner, A. Baliva, and S. Di Lorenzo, Morphofacies analysis and depositional architecture of the Eberswalde crater, Mars, Icarus, doi:10.1016/j.icarus.2008.05.018, 2008.

Rossi, A. P., S. van Gasselt, M. Pondrelli, T. Zegers, E. Hauber, and G. Neukum, Periglacial landscape evolution at lower mid-latitudes on Mars: The Thaumasia highlands, Ninth International Conference on Permafrost Proceedings, 1531-1536, 2008.

Scholten, F., K. Gwinner, T. Roatsch, K.-D. Matz, M. Wahlisch, B. Giese, J. Oberst, R. Jaumann, and G. Neukum, Mars express HRSC data processing - methods and operational aspects, Photogramm. Eng. Rem. S., 71(10), 1143-1153, 2005.

Spiegel, M., Improvement of interior and exterior orientation of the three line camera HRSC with a simultaneous adjustment, Inter. Arch. of Photogramm. Eng. Rem. S, 36(3/W49B), 161-166, 2007.

Smith, D. E., M. T. Zuber, H. V. Frey, J. B. Garvin, J. W. Head, D. O. Muhleman, G. H. Pettengill, R. J. Phillips, S. C. Solomon, H. J. Zwally, W. B. Banerdt, T. C. Duxbury, M. P. Golombek, F. G. Lemoine, G. A. 
Neumann, D. D. Rowlands, O. Aharonson, P. G. Ford, A. B. Ivanov, P. J. McGovern, J. B. Abshire, R. S. Afzal, and X. Sun, Mars orbiter laser altimeter: Experiment summary after the first year of global mapping of Mars, J. Geophys. Res., 106(E10), 23689-23722, 2001.

Warner, N., S. Gupta, J.-P. Muller, J.-R. Kim, and S.-Y. Lin, A refined chronology of catastrophic outflow events in Ares Vallis, Mars, Earth Planet. Sci. Lett., 288(1-2), 58-69, 2009.

Warner, N., S. Gupta, J.-R. Kim, S.-Y. Lin, and J.-P. Muller, Hesperian equatorial thermokarst lakes in Ares Vallis as evidence for transient warm conditions on Mars, Geology, 38(1), 71-74, 2010a.

Warner, N., S. Gupta, J.-R. Kim, S.-Y. Lin, and J.-P, Muller, Retreat of a giant cataract in a long-lived $(3.7 \mathrm{Ga}-2.6 \mathrm{Ga})$ martian outflow channel, Geology, 38(9), 791-794, 2010b.
Warner, N., S. Gupta, S.-Y. Lin, J.-R. Kim, J.-P. Muller, and J. Morley, Late Noachian to Hesperian climate change on Mars: Evidence of episodic warming from transient crater lakes near Ares Vallis, J. Geophys. Res., 115, E06013, doi:10.1029/2009JE003522, 2010c.

Warner, N. H., S. Gupta, J.-R. Kim, J.-P. Muller, L. Le Corre, J. Morley, S.-Y. Lin, and C. McGonigle, Constraints on the origin and evolution of Iani Chaos, Mars, J. Geophys. Res., 116, E06003, doi:10.1029/2010JE003787, 2011.

J.-R. Kim (e-mail: kjrr001@gmail.com), S.-Y. Lin, Y.-S. Choi, and Y.H. Kim 